\title{
Effect of Switching from Cilnidipine to Azelnidipine on Cardiac Sympathetic Nerve Function in Patients with Heart Failure Preserved Ejection Fraction
}

\author{
Shunsuke Kiuchi, ${ }^{1}$ MD, Shinji Hisatake, ${ }^{1}$ MD, Takayuki Kabuki, ${ }^{1}$ MD, Takashi Oka, ${ }^{1}$ MD, \\ Shintaro Dobashi, ${ }^{1}$ MD, Takahiro Fujii, ${ }^{1}$ MD and Takanori Ikeda, ${ }^{1}$ MD
}

\begin{abstract}
Summary
Cardiac sympathetic nerve activity is known to play a key role in the development and progression of heart failure (HF). Azelnidipine, an L-type calcium channel blocker (CCB), inhibits the sympathetic nerve activity of the central system. In contrast, cilnidipine, an N-type CCB, inhibits the sympathetic nerve activity of the peripheral system. CCBs are recommended as class IIa in patients with HF preserved ejection fraction (HFpEF); however, there are no comparative data on the difference in effect of cilnidipine and azelnidipine in patients with HFpEF and hypertension. We investigated the difference in effect of azelnidipine compared with cilnidipine in patients with HFpEF. Twenty-four consecutive HF patients who received angiotensin II type1a receptor blocker and beta blocker from April 2013 to January 2015 were enrolled. Cilnidipine was switched to azelnidipine during the follow-up period. Blood pressures, heart rate, blood tests, echocardiography, and ${ }^{123} \mathrm{I}-$ metaiodobenzylguanidine (MIBG) cardiac-scintigraphy were measured before and after 6 months from azelnidipine administration. B-type natriuretic peptide tended to decrease after switching to azelnidipine; however, there were no significant differences between the pre-state and post-state (pre-state: $118.5 \mathrm{pg} / \mathrm{mL}$ and post-state: $78.4 \mathrm{pg} / \mathrm{mL}, P=0.137$ ). Other laboratory findings, including catecholamine, also did not change significantly. In echocardiography, there were no significant differences in systolic and diastolic functions at the pre-state and post-state. As for MIBG, there were no significant changes in heart/mediastinum ratio. However, washout rate was significantly reduced (pre-state: 42.9 and post-state: $39.6, P=0.030$ ). Azelnidipine improved the dysfunction of cardiac sympathetic nerve activity compared with cilnidipine in patients with HFpEF.
\end{abstract}

(Int Heart J 2018; 59: 120-125)

Key words: Cardiac sympathetic nerve activity, ${ }^{123}$ I-metaiodobenzylguanidine Cardiac-scintigraphy

$\mathrm{S}$ ome previous studies revealed that cardioprotective effect of medications, such as beta blocker (BB) and renin-angiotensin-aldosterone system inhibitor (RAAS-I) (angiotensin-converting enzyme inhibitor and angiotensin II type 1a receptor blocker (ARB)). Those medications improved the prognosis of heart failure (HF) reduced ejection fraction $(\mathrm{EF})(\mathrm{HFrEF}){ }^{1-3)}$ On the other hand, the cardioprotective effect of medications for HF preserved $\mathrm{EF}$ (HFpEF) is still not established. In the Japanese Circulation Society therapeutic guideline of chronic $\mathrm{HF}$, these medications are recommended as class IIa in patients with HFpEF. These medications also have antihypertensive effects. Therefore, majority of patients treated with these medications have normotension or hypotension. However, some patients still have hypertension (HT); thus, those patients are usually treated with antihypertensive medications, including calcium channel blockers (CCBs).

Dihydropyridine $\mathrm{CCB}$ is classified to $\mathrm{L}, \mathrm{N}$, and $\mathrm{T}$ subtypes. ${ }^{4)}$ L-type CCBs have strict antihypertensive effects although reflectivity on sympathetic nerve hyperactivity could appear. Azelnidipine, a unique long-acting Ltype CCB, cancels the reflectivity of sympathetic nerve hyperactivity, which suppresses the sympathetic nerve activity of the central system through the rostral ventrolateral medulla (RVLM) ${ }^{5,6)}$ It had also been reported that central sympathetic hyperactivity through RVLM is associated with $\mathrm{HT}^{7)}$ Cilnidipine, an L- and N-type CCB, also improves this reflectivity of sympathetic nerve hyperactivity. ${ }^{8)}$ The excessive release of noradrenaline from the sympathetic nerve ending induces this effect. ${ }^{9}$

There are no comparative data on the differences in adding cilnidipine or azelnidipine to BB and RAAS-I in patients with HFpEF and HT.

From the ${ }^{1}$ Department of Cardiovascular Medicine, Toho University Faculty of Medicine, Tokyo, Japan.

This manuscript was supported in part by Grants-in-Aid (24591074 and 15K09103 to T.I.) for Scientific Research from the Ministry of Education, Culture, Sports, Science and Technology of Japan.

Address for correspondence: Shunsuke Kiuchi, MD, Department of Cardiovascular Medicine, Toho University Graduate School of Medicine, 6-11-1 Omorinishi, Ota-ku, Tokyo 143-8541, Japan. E-mail: syunnsuke@med.toho-u.ac.jp

Received for publication January 14, 2017. Revised and accepted March 23, 2017

Released in advance online on J-STAGE December 20, 2017.

doi: 10.1536/ihj.17-024

All rights reserved by the International Heart Journal Association. 


\section{Methods}

The present study is approved by the ethics committee of Toho University Hospital (approval number: 24213). All experiments were performed in accordance with the Declaration of Helsinki. The present study protocol was a single-center, single-design open prospective study.

Study subjects: Twenty-four consecutive chronic HFpEF patients with HT who consulted our institute from April 2013 to January 2015 were enrolled. Almost all patients have received $\mathrm{ARB}, \mathrm{BB}$, or $\mathrm{CCB}$ for more than 3 years and had a stable condition of chronic HF. Initially, we administrated cilnidipine for HT. If patients had been administrated other $\mathrm{CCB}$ than cilnidipine, $\mathrm{CCB}$ was changed to cilnidipine and was administered for more than 4 weeks. Then, we changed to azelnidipine from cilnidipine. The dosage of cilnidipine and azelnidipine were adjusted in accordance with the targets of HT management from the Japanese Society of HT. The following findings of before azelnidipine administration were compared with at 6 months after switching to azelnidipine. These findings were evaluated on the same day. The exclusion criteria were (1) age under 30 or over 90 , (2) past history of receiving cilnidipine and/or azelnidipine, (3) subjects undergoing hemodialysis, (4) subjects suspected of having Parkinson's disease, and (5) subjects with phaeochromocytoma or malignant neoplasma.

Blood pressures and heart rates: We measured blood pressures (BPs) twice with an aneroid sphygmomanometer after the participants had been comfortably seated for at least 5 minutes and calculated these average. ${ }^{10)}$ Moreover, pulse pressure (PP) and mean blood pressure (mBP) were also calculated, using the following formula: $\mathrm{PP}=$ systolic $\mathrm{BP})$ - diastolic blood pressure $(\mathrm{dBP})$ and $\mathrm{mBP}=\mathrm{dBP}+$ $\mathrm{PP} / 3$, respectively. We evaluated heart rate $(\mathrm{HR})$ at the same time by standard 12-lead electrocardiography (ECG). ECG was performed after the patients had been kept in the resting position.

Laboratory analysis: As for lipid metabolism markers. We evaluated total cholesterol (T-Cho), triglyceride (TG), high-density lipoprotein cholesterol (HDL), low-density lipoprotein cholesterol (LDL), and LDL/HDL ratio. LDL was calculated with the Friedewald formula: $\mathrm{LDL}=\mathrm{T}$ Cho - HDL - TG/5. ${ }^{11)}$ We also evaluated fasting plasma glucose and hemoglobin A1C as glucose metabolism markers. Liver functions (aspartate aminotransferase, alanine aminotransferase, and lactate dehydrogenase) and renal functions (blood urea nitrogen, creatinine, and estimated glomerular filtration rate (eGFR)) were evaluated. We calculated the eGFR from the Japanese Society of Nephrology criteria: eGFR $=194 \times \mathrm{Cr}^{-1.094} \times$ age $^{-0.287}($ for men) and $194 \times \mathrm{Cr}^{-1.094} \times$ age $^{-0.287} \times 0.739$ (for women). ${ }^{12)}$ Moreover, we evaluated brain natriuretic peptide (BNP) as the assessment of the severity of HF. Epinephrine, norepinephrine (NE), and dopamine were evaluated as the systemic sympathetic nerve parameters. All serum samples were obtained in the fast state after resting in the supine position for at least 5 minutes.

Transthoracic echocardiography: Transthoracic echocardiography was evaluated by two cardiologists. Cardiologists were blinded and evaluated both examinations (pre- state and post-state). Left ventricular (LV) diameter of diastolic/systolic and LV wall thickness (interventricular septum thickness and posterior LV wall thickness) from parasternal long-axis view were investigated. We investigated LV systolic function from EF and \%fractional shortening with the Teichholz method ${ }^{13)}$ by parasternal longaxis view or modified Simpson's method ${ }^{14)}$ by apical twoor four-chamber view. HFpEF was defined as an EF $\geqq$ $50 \% .{ }^{15)}$ Left atrial diameter was evaluated from long and short axes of the apical four-chamber view. Peak velocities of early (E) and late (A) filling from the mitral valve Doppler echocardiography were evaluated, and we calculated the E/A ratio. We investigated LV diastolic function from the E/A ratio. In the mitral and aortic valve Doppler echocardiography, we also evaluated the Tei index.

Cardiac-scintigraphy: Cardiac sympathetic nerve activity was investigated by cardiac-scintigraphy using ${ }^{123} \mathrm{I}-$ metaiodobenzylguanidine (MIBG). Each of the subjects was given ${ }^{123} \mathrm{I}-\mathrm{MIBG}$ intravenously. Anterior planar images were obtained at 20 minutes (early images) and 4 hours (late images) after the intravenous injection. The average of heart region of interest $(\mathrm{ROI})(\mathrm{H})$ to the average of mediastinum ROI (M) ratio (H/M ratio) in early and late planar images was calculated. In addition, washout rate (WR) was calculated with the formula: WR $(\%)=($ early image $(\mathrm{H}-\mathrm{M})$ - late image $(\mathrm{H}-\mathrm{M}))$ /early image $(\mathrm{H}-\mathrm{M}) \times$ $100 .{ }^{16)}$

Statistical analysis: We set the difference and the standard deviation (S.D.) of BPs that changed by switching cilnidipine to azelnidipine as 10 and 16.8 , respectively. The number of subjects required for valid statistical analysis was calculated to 23 under the conditions $\alpha=0.05$ and power $=0.8$. Therefore, 24 consecutive patients with HFpEF were enrolled. Continuous variables were expressed as the mean \pm S.D. or the median. The Wilcoxon signed-rank test was used to compare pre-state and poststate. Analyses were performed with Microsoft Excel and the Stat View statistical package (Stat View 4.0, SAS Institute Inc.). A probability $(P)$ value of less than 0.05 was considered to indicate statistical significance.

\section{Results}

Patient characteristics: There were 17 male patients $(70.8 \%$ ), and the average age was 73.0 years (43 to 85 years). In the present study, subjects had various etiologies of HF (Table I). Patients with HFrEF and HFpEF at the first onset of HF were $9(37.5 \%)$ and $15(62.5 \%)$, respectively. ARB had been administered to $100 \%$ of the patients. BB was unable to be used for three patients, because of low HR or bronchial asthma. Therefore, BB had been administered to $21(87.5 \%)$ patients. The average dosage of cilnidipine and azelnidipine were $14.3 \pm 3.32$ and $8.2 \pm 5.3 \mathrm{mg}$, respectively.

Changes in blood pressures and heart rate: Table II shows changes in BPs, HR, and laboratory findings. BPs did not statistically change between pre-state and poststate. HR tended to decrease after switching to azelnidipine; however, there were no significant differences between the pre-state and post-state.

Changes in laboratory findings: Laboratory findings are 
Table I. Underlying Heart Disease and Medications

\begin{tabular}{lc}
\hline & $n, \%$ \\
\hline Dilated cardiomyopathy & $2,8.3 \%$ \\
Hypertrophic cardiomyopathy & $1,4.2 \%$ \\
Ischemic cardiomyopathy & $11,45.8 \%$ \\
Hypertensive heart disease & $4,16.7 \%$ \\
Tachycardia induced cardiomyopathy & $3,12.5 \%$ \\
Valvular heart disease & $3,12.5 \%$ \\
HFrEF (at the first onset of heart failure) & $9,37.5 \%$ \\
HFpEF (at the first onset of heart failure) & $15,62.5 \%$ \\
Angiotensin converting enzyme inhibitor & $0,0 \%$ \\
Angiotensin II type 1a receptor blocker & $24,100 \%$ \\
B-blocker & $21,87.5 \%$ \\
Carvedilol & $16,66.7 \%$ \\
Bisoprolol & $5,20.8 \%$ \\
$\alpha$-blocker & $0,0 \%$ \\
Diuretics & $9,37.5 \%$ \\
HMG-CoA reductase inhibitor & $16,66.7 \%$ \\
\hline
\end{tabular}

HFrEF indicates heart failure reduced ejection fraction; $\mathrm{HFpEF}$, heart failure preserved ejection fraction; and HMG-CoA reductase inhibitor, Hydroxymethylglutaryl$\mathrm{CoA}$ reductase inhibitor

Table II. Changes in Blood Pressures, Heart Rates, and Laboratory Findings

\begin{tabular}{lccc}
\hline & Pre-state & Post-state & $P$-value \\
\hline sBP (mmHg) & 134.0 & 145.0 & 0.324 \\
dBP (mmHg) & 84.0 & 85.0 & 0.715 \\
PP (mmHg) & 51.5 & 57.0 & 0.172 \\
mBP (mmHg) & 99.8 & 103.2 & 0.483 \\
HR (bpm) & 64.0 & 56.5 & 0.386 \\
AST (IU/L) & 26.0 & 25.0 & 0.773 \\
ALT (IU/L) & 20.5 & 16.5 & 0.952 \\
LDH (IU/L) & 217.5 & 221.5 & 0.230 \\
BUN (mg/dL) & 22.0 & 20.5 & 0.654 \\
Creatinine (mg/dL) & 1.14 & 1.14 & 0.875 \\
eGFR (mL/minute/1.73m $\left.{ }^{2}\right)$ & 47.7 & 51.5 & 0.627 \\
T-Cho (mg/dL) & 168.8 & 176.1 & 0.290 \\
TG (mg/dL) & 102.5 & 98.0 & 0.943 \\
HDL (mg/dL) & 51.0 & 53.5 & 0.399 \\
LDL (mg/dL) & 90.5 & 98.5 & 0.432 \\
LDL/HDL ratio & 1.747 & 1.884 & 0.997 \\
FPG (g/dL) & 111.5 & 115.5 & 0.214 \\
HbA1C (\%) & 5.80 & 5.90 & 0.904 \\
Hemoglobin (g/dL) & 12.8 & 12.7 & 0.338 \\
Epinephrine (ng/mL) & 0.03 & 0.04 & 0.904 \\
Nor-epinephrine (ng/mL) & 0.48 & 0.57 & 0.549 \\
Dopamine (ng/mL) & 0.02 & 0.02 & 0.824 \\
BNP (pg/mL) & 118.5 & 78.4 & 0.137 \\
\hline
\end{tabular}

sBP indicates systolic blood pressure; $\mathrm{dBP}$, diastolic blood pressure; PP, pulse pressure; $\mathrm{mBP}$, mean blood pressure; HR, heart rate; AST, aspartate aminotransferase; ALT, alanine aminotransferase; $\mathrm{LDH}$, lactate dehydrogenase; BUN, blood urea nitrogen; eGFR, estimated glomerular filtration rate; T-Cho, total cholesterol; TG, triglyceride; HDL, high-density lipoprotein cholesterol; LDL, low-density lipoprotein cholesterol; FPG, fasting plasma glucose; $\mathrm{HbA1C}$, hemoglobin $\mathrm{A} 1 \mathrm{C}$; and $\mathrm{BNP}$, brain natriuretic peptide. Continuous data are expressed the median. $P$-values were determined using the Wilcoxon signed-rank test.

shown in Table II. There were no significant changes even though the patients had mild renal dysfunction at the time
Table III. Changes in Transthoracic Echocardiography

\begin{tabular}{lccc}
\hline & Pre-state & Post-state & $P$-value \\
\hline LAD (long axis: $\mathrm{mm})$ & 48.8 & 53.2 & 0.274 \\
LAD (short axis: mm) & 41.5 & 44.3 & 0.267 \\
LVDd (mm) & 51.8 & 50.2 & 0.198 \\
LVDs (mm) & 32.8 & 30.9 & 0.853 \\
IVST (mm) & 9.36 & 9.60 & 0.808 \\
PWT (mm) & 9.73 & 10.10 & 0.976 \\
EF $(\%)$ & 68.4 & 67.6 & 0.721 \\
\%FS $(\%)$ & 38.4 & 37.9 & 0.721 \\
E/A & 0.692 & 0.731 & 0.807 \\
TEI index & 0.467 & 0.410 & 0.184 \\
\hline
\end{tabular}

LAD indicates left atrial diameter; LVDd, left ventricular diameter diastolic; LVDs, left ventricular diameter systolic; IVST, interventricular septum thickness; PWT, posterior LV wall thickness; EF, ejection fraction; \%FS, \%fractional shortning; and E/A, E wave/A wave ratio. Continuous data are expressed the median. $P$-values were determined using the Wilcoxon signed-rank test.

of study enrollment. Liver functions also did not change significantly. Lipid and glucose metabolisms were not affected from switching to azelnidipine. BNP, which indicates the severity of HF, tended to decrease after switching to azelnidipine; however, there were no significant differences between the pre-state and post-state (pre-state: $118.5 \mathrm{pg} / \mathrm{mL}$ and post-state: $78.4 \mathrm{pg} / \mathrm{mL}, P=0.137)$. The systemic sympathetic nerve parameters also did not significantly changed after switching to azelnidipine.

The improvement of left ventricular diastolic function in transthoracic echocardiography: Table III shows that there were no significant differences in left atrial/ventricular diameter and LV wall thickness. In addition, LV function evidenced with TEI index has no statistical difference between the pre-state and post-state. In LV systolic and diastolic functions, there were also no significant changes after switching to azelnidipine.

Changes in cardiac sympathetic nerve activity with cardiac-scintigraphy: The primary endpoint in the present study was the evaluation of cardiac sympathetic nerve activity using ${ }^{123}$ I-MIBG cardiac-scintigraphy. The changes in $\mathrm{H} / \mathrm{M}$ ratio (early and late images) with ${ }^{123} \mathrm{I}-\mathrm{MIBG}$ are shown in Figure A. There were no statistical changes in $\mathrm{H} / \mathrm{M}$ ratios. WR with ${ }^{123} \mathrm{I}-\mathrm{MIBG}$ in the post-state was significantly decreased compared with the pre-state, indicating amelioration of cardiac sympathetic nerve hyperactivity (pre-state: 42.9 and post-state: $39.6, P=0.030$, Figure B).

\section{Discussion}

Main findings: Previous study also revealed that azelnidipine treatment had a tendency to decrease HR compared with cilnidipine treatment in patients with type 2 diabetes mellitus. ${ }^{17)}$ In the present study, HR also tended to decrease by switching to azelnidipine. In addition, WR in ${ }^{123}$ I-MIBG significantly decreased by azelnidipine treatment, compared with cilnidipine treatment. These results suggested that cardiac sympathetic nerve hyperactivity was improved by azelnidipine treatment, compared with cilnidipine treatment. On the other hand, the systemic 

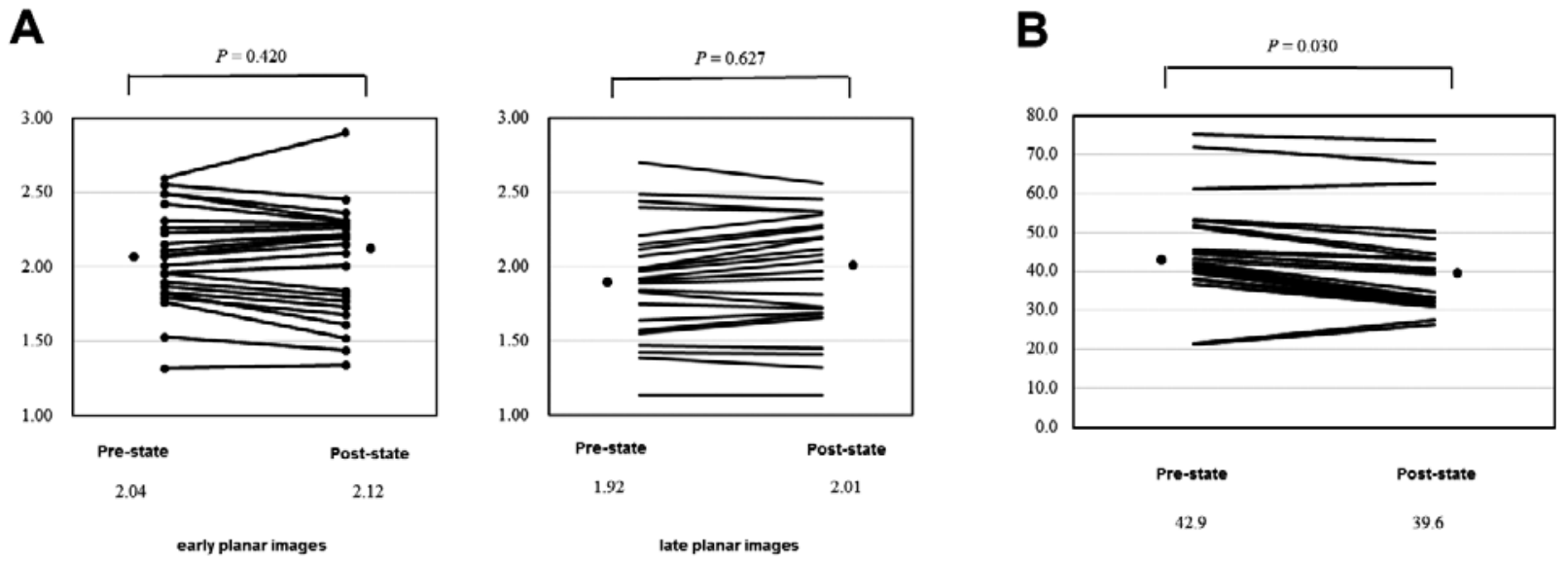

Figure. Comparison of ${ }^{123}$ I-metaiodobenzylguanidine (MIBG) cardiac-scintigraphy findings between the pre-state and post-state. In MIBG, there was no change in $\mathrm{H} / \mathrm{M}$ ratios (A) in early and late images. However, washout rate (B) was significantly reduced. Continuous data are expressed as the median $P$-values were determined using the Wilcoxon signed-rank test.

sympathetic nerve parameters did not change significantly. Medications for heart failure: RAAS-I and BB are established treatments of HFrEF. In addition, these medications are recommended as class IIa in HFpEF patients by the Japanese Circulation Society therapeutic guideline for chronic HF. In the present study, nine patients had HFrEF at the first onset of acute HF and HFpEF at the time of study entry. The optimal choice of medications when EF was improved is also remaining unclear. Therefore, we enrolled patients treated with RAAS-I and BB, in the present study. On the other hand, the guidelines for the management of HT from the Japanese Society of HT recommend RAAS-I, BB, CCB, and diuretics (thiazide) as the first choice of antihypertensive medications. ${ }^{10)}$ In HF, generally, diuretics may be used when the body fluid volume was considered. CCB is recommended as class IIa in patients with HFpEF. In addition, it has also been reported that combined use of $\mathrm{CCB}$ and $\mathrm{ARB}$ is useful for visit-tovisit variability and seasonal variation in $\mathrm{BP}^{18)}$ In the present study, azelnidipine inhibited cardiac sympathetic nerve activity more strongly compared with cilnidipine, even under $\mathrm{ARB}$ and $\mathrm{BB}$ administration. The present study showed the different effect of azelnidipine and cilnidipine on sympathetic nerve activity. Azelnidipine inhibits vascular calcification in addition to the effects of blocking calcium channel. ${ }^{19)}$ On the other hand, cilnidipine has renal protective effects by dilating the import and export artery and improves early morning HT. ${ }^{20,21)}$ It is necessary to give considerations on the above points when making a decision for medications.

${ }^{123}$ I-MIBG cardiac-scintigraphy and cardiac sympathetic nerve activity: ${ }^{123} \mathrm{I}-\mathrm{MIBG}$, an analog of NE, is associated with myocardial NE in HF patients. ${ }^{22)}$ Therefore, cardiac sympathetic hyperactivity evaluated by ${ }^{123}$ I-MIBG cardiac-scintigraphy is associated with the evaluation of severity and the prognosis for $\mathrm{HF}^{23,24)}$ It has been reported that WR was a useful parameter for the prediction of the prognosis of HF compared with delayed $\mathrm{H} / \mathrm{M}$ ratio, ${ }^{25)}$ although WR and delayed H/M ratio are both useful. ${ }^{26)}$ Moreover, elevated WR was related with sudden cardiac death in $\mathrm{HF}^{27)}$ In the present study, azelnidipine signifi- cantly reduced WR. On the other hand, the systemic sympathetic nerve parameters investigated with the laboratory data did not change significantly. It has been reported that cardiac sympathetic nerve in MIBG has improved prior to the improvement of the systemic sympathetic nerve in HF patients. ${ }^{28)}$ These results suggest that for the treatment of HFpEF, azelnidipine is superior to cilnidipine in improving cardiac sympathetic nerve function that leads to amelioration of the prognosis of HF.

Heart failure and cardiac sympathetic nerve activity: Cardiac sympathetic nerve hyperactivity enhances the severity of HF. On the other hand, the exacerbation of $\mathrm{HF}$ enhances cardiac sympathetic nerve function. In the present study, there are significant increases in WR, indicating the cardiac sympathetic nerve hyperactivity. However, BNP, which indicates the severity of HF, did not change. Therefore, it was considered that the improvement of cardiac sympathetic nerve function ameliorates the severity of HF. Azelnidipine inhibits cardiac sympathetic nerve hyperactivity of the central system, whereas cilnidipine has inhibitory action of cardiac sympathetic nerve hyperactivity in the peripheral. The difference of inhibitory effects of cardiac sympathetic nerve function between azelnidipine and cilnidipine in the present study is thought to be due to these mechanisms. Elevated HR were associated with the worsening prognosis of HF in the CHART-2 study, ${ }^{29)}$ although HR is known to be associated with sympathetic nerve activity. In the present study, HR also tended to decrease by switching to azelnidipine.

Study limitations: Study protocol was a single-center, single-arm, open prospective study, because the number of target study populations was extremely small. We could not conduct crossover and/or randomized trials; therefore, the study design is the most important limitation in the present study. Nine patients have $\mathrm{HFpEF}$ at the time of the study entry and have HFrEF at the first onset of acute HF. It has not been revealed that we have an optimal choice of treatment for patients with continuous HFpEF or patients who have HFpEF migrated from HFrEF. In the present study, the number of subjects was not enough to evaluate and investigate these in detail. It is necessary to 
reveal these results in future research with a large-scale prospective study carried with either crossover design or randomized control trial.

\section{Conclusions}

This small-scale study demonstrated that azelnidipine improved cardiac sympathetic nerve activity compared with cilnidipine in HF patients with essential HT. Amelioration of cardiac sympathetic nerve activity may lead to the improvement in prognosis of HF. Large-scale clinical studies are necessary to confirm these results.

\section{Disclosures}

Conflicts of interest: T.I. received research funds and lecture fees from Mitsubishi Tanabe Pharma Co., Ltd., Daiichi-Sankyo, Co., Ltd., and Ono Pharmaceutical, Co., Ltd. The remaining authors declare that there is no conflict of interest.

\section{References}

1. Nitta D, Kinugawa K, Imamura T, Kato NP, Komuro I. High dose $\beta$-blocker therapy triggers additional reverse remodeling in patients with idiopathic non-ischemic cardiomyopathy. Int Heart J 2016; 57: 717-24.

2. Ahn SA, Jong P, Yusuf S, Bangdiwala SI, Pouleur HG, Rousseau MF. Early versus delayed enalapril in patients with left ventricular systolic dysfunction: impact on morbidity and mortality 15 years after the SOLVD trial. J Am Coll Cardiol 2006 47: 1904-5

3. Ohsaka T, Inomata T, Naruke T, et al. Clinical impact of adherence to guidelines on the outcome of chronic heart failure in $\mathrm{Ja}$ pan. Int Heart J 2008; 49: 59-73.

4. Catterall WA, Striessnig J. Receptor sites for $\mathrm{Ca} 2+$ channel antagonists. Trends Pharmacol Sci 1992; 13: 256-62.

5. Kimura Y, Hirooka Y, Sagara Y, Sunagawa K. Long-acting calcium channel blocker, azelnidipine, increases endothelial nitric oxide synthase in the brain and inhibits sympathetic nerve activity. Clin Exp Hypertens 2007; 29: 13-21.

6. Ogawa K, Hirooka Y, Shinohara K, Kishi T, Sunagawa K. Inhibition of oxidative stress in rostral ventrolateral medulla improves impaired baroreflex sensitivity in stroke-prone spontaneously hypertensive rats. Int Heart J 2012; 53: 193-8.

7. Kishi T. Heart Failure as a disruption of dynamic circulatory homeostasis mediated by the brain. Int Heart J 2016; 57: 145-9.

8. Tanaka M, Sekioka R, Nishimura T, Ichihara A, Itoh H. Effects of cilnidipine on sympathetic nerve activity and cardiorenal function in hypertensive patients with type 2 diabetes mellitus: association with BNP and aldosterone levels. Diabetes Res Clin Pract 2014; 106: 504-10.

9. Fujii S, Kameyama K, Hosono M, Hayashi Y, Kitamura K. Effect of cilnidipine, a novel dihydropyridine $\mathrm{Ca}++-$ channel antagonist, on $\mathrm{N}$-type $\mathrm{Ca}++$ channel in rat dorsal root ganglion neurons. J Pharmacol Exp Ther 1997; 280: 1184-91.

10. Shimamoto K, Ando K, Fujita T, et al. The Japanese society of hypertension guidelines for the management of hypertension (JSH 2014). Hypertens Res 2014; 37: 253-390.

11. Friedewald WT, Levy RI, Fredrickson DS. Estimation of the concentration of low-density lipoprotein cholesterol in plasma, without use of the preparative ultracentrifuge. Clin Chem 1972; 18: 499-502.

12. Matsuo S, Imai E, Horio M, et al. Revised equations for estimated GFR from serum creatinine in Japan. Am J Kidney Dis
2009; 53: 982-92.

13. Teichholz LE, Kreulen T, Herman MV, Gorlin R. Problems in echocardiographic volume determinations: echocardiographicangiographic correlations in the presence or absence of asynergy. Am J Cardiol 1976; 37: 7-11.

14. Schiller NB, Shah PM, Crawford M, et al. Recommendations for quantitation of the left ventricle by two-dimensional echocardiography. American Society of Echocardiography Committee on Standards, Subcommittee on Quantitation of TwoDimensional Echocardiograms. J Am Soc Echocardiogr 1989; 2: 358-67.

15. Marui A, Nishiwaki N, Komiya T, et al. Comparison of 5-year outcomes after coronary artery bypass grafting in heart failure patients with versus without preserved left ventricular ejection fraction (from the CREDO-Kyoto CABG Registry Cohort-2). Am J Cardiol 2015; 116: 580-6.

16. Hisatake S, Nanjo S, Fujimoto S, et al. Comparative analysis of the therapeutic effects of long-acting and short-acting loop diuretics in the treatment of chronic heart failure using (123)Imetaiodobenzylguanidine scintigraphy. Eur J Heart Fail 2011; 13: $892-8$

17. Abe H, Mita T, Yamamoto R, et al. Comparison of effects of cilnidipine and azelnidipine on blood pressure, heart rate and albuminuria in type 2 diabetics with hypertension: A pilot study. $\mathrm{J}$ Diabetes Investig 2013; 4: 202-5.

18. Sato N, Saijo Y, Sasagawa Y, et al. Visit-to-visit variability and seasonal variation in blood pressure: Combination of Antihypertensive Therapy in the Elderly, Multicenter Investigation (CAMUI) Trial subanalysis. Clin Exp Hypertens 2015; 37: 411-9.

19. Shimizu T, Tanaka T, Iso T, Kawai-Kowase K, Kurabayashi M. Azelnidipine Inhibits Msx2-dependent osteogenic differentiation and matrix mineralization of vascular smooth muscle cells. Int Heart J 2012; 53: 331-5.

20. Konno Y, Kimura K. Vasodilatory Effect of Cilnidipine, an Ltype and N-type calcium channel blocker on rat kidney glomerular arterioles. Int Heart J 2008; 49: 723-32.

21. Ashizawa N, Seto S, Shibata Y, Yano K. Bedtime administration of cilnidipine controls morning hypertension. Int Heart J 2007; 48: 597-603.

22. Schofer J, Spielmann R, Schuchert A, Weber K, Schluter M. Iodine-123 meta-indobenzylguanidine scintigraphy: a noninvasive method to demonstrate myocardial adrenergic nervous system disintegrity in patients with idiopathic dilated cardiomyopathy. J Am Coll Cardiol 1988; 12: 1252-8.

23. Kasama S, Toyama T, Kurabayashi M. Usefulness of cardiac sympathetic nerve imaging using 123 iodinemetaiodobenzylguanidine scintigraphy for predicting sudden cardiac death in patients with heart failure. Int Heart J 2016; 57: 140-4.

24. Narula J, Gerson M, Thomas GS, Cerqueria MD, Jacobson AF. 123I-MIBG Imaging for prediction of mortality and potentially fatal events in heart failure: The ADMIRE-HFX Study. J Nucl Med 2015; 56: 1011-8.

25. Kuwabara Y, Tamaki N, Nakata T, Yamashina S, Yamazaki J. Determination of the survival rate in patients with congestive heart failure stratified by 123I-MIBG imaging: a meta-analysis from the studies performed in Japan. Ann Nucl Med 2011; 25: 101-7.

26. Kinbara T, Hayano T, Otani N, Furutani Y, Tanaka S. Iodine-123 metaiodobenzylguanidine imaging can predict future cardiac events in Japanese patients with Parkinson's disease. Ann Nucl Med 2013; 27: 123-31.

27. Kasama S, Toyama T, Kaneko Y, et al. Relationship between late ventricular potentials and myocardial $123 \mathrm{I}-$ metaiodobenzylguanidine scintigraphy in patients with dilated cardiomyopathy with mild to moderate heart failure: results of a prospective study of sudden death events. Eur J Nucl Med Mol Imaging 2012; 39: 1056-64.

28. Tsutamoto T, Tanaka T, Sakai H, et al. Beneficial effect of perindopril on cardiac sympathetic nerve activity and brain natriu- 
retic peptide in patients with chronic heart failure: comparison with enalapril. Circ J 2008; 72: 740-6.

29. Takada T, Sakata Y, Miyata S, et al. Impact of elevated heart rate on clinical outcomes in patients with heart failure with reduced and preserved ejection fraction: a report from the CHART-2 Study. Eur J Heart Fail 2014; 16: 309-16. 